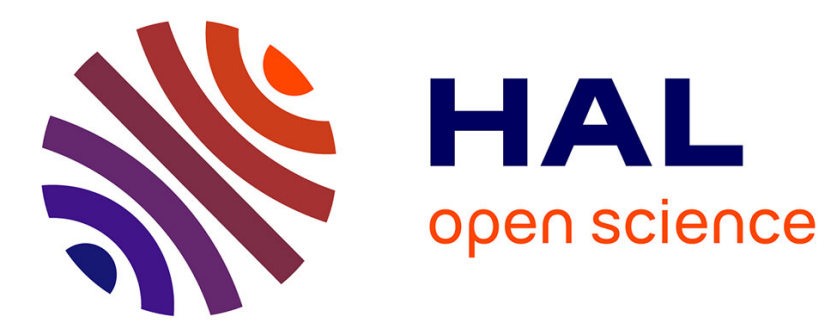

\title{
The MBR intertemporal choice criterion and Rawls' just savings principle
}

Charles C. Figuieres, Ngo van Long, Mabel Tidball

\section{To cite this version:}

Charles C. Figuieres, Ngo van Long, Mabel Tidball. The MBR intertemporal choice criterion and Rawls' just savings principle. 2013. hal-02809724

\section{HAL Id: hal-02809724 \\ https://hal.inrae.fr/hal-02809724}

Preprint submitted on 6 Jun 2020

HAL is a multi-disciplinary open access archive for the deposit and dissemination of scientific research documents, whether they are published or not. The documents may come from teaching and research institutions in France or abroad, or from public or private research centers.
L'archive ouverte pluridisciplinaire HAL, est destinée au dépôt et à la diffusion de documents scientifiques de niveau recherche, publiés ou non, émanant des établissements d'enseignement et de recherche français ou étrangers, des laboratoires publics ou privés. 


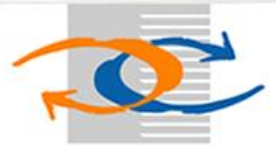

\section{A M E T A}

Laboratoire Montpelliérain d'Economie Théorique et Appliquée

U M R

Unité Mixte de Recherche

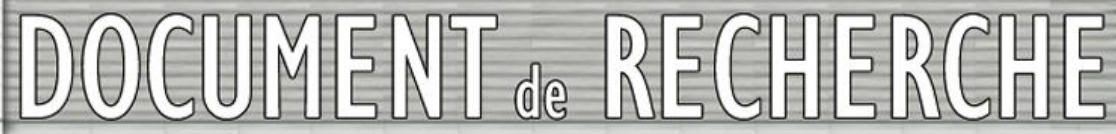

« The MBR Intertemporal Choice Criterion and Rawls' Just Savings Principle »

Charles FIGUIËRES

Ngo Van LONGY

Mabel TIDBALL

DR $n^{\circ} 2013-02$

Unite de Formation et de Recherche d'Economie Avenue Raymond DUGRAND C.S. 79606

34960 MONTPELLIER Cedex 2

E-mail : lameta@lameta.univ-montp1.fr web : www.lameta.univ-montp1.fr

\section{U, 1}




\title{
The MBR Intertemporal Choice Criterion and Rawls' Just Savings Principle
}

\author{
Charles Figuières, Ngo Van Long ${ }^{\dagger}$ and Mabel Tidball ${ }^{\ddagger}$
}

February 20, 2013

\begin{abstract}
This paper provides general theorems about the control that maximizes the mixed Bentham-Rawls (MBR) criterion for intergenerational justice, which was introduced in Alvarez-Cuadrado and Long (2009). We establish sufficient concavity conditions for a candidate trajectory to be optimal and unique. We also show that the state variable is monotonic under rather weak conditions. And finally we prove that inequality among generations, captured by the gap between the poorest and the richest generations, is lower when optimization is performed under the MBR criterion rather than under the discounted utilitarian criterion. The two last properties are in line with some aspects of the rawlsian just savings principle.
\end{abstract}

Keywords: intergenerational equity, just savings principle.

JEL Classification: D63, H43, O21, Q20.

*INRA-LAMETA, 2 Place Viala, 34060 cedex 1. Montpellier. France. Email: Charles.Figuieres@supagro.inra.fr.

${ }^{\dagger}$ Department of Economics, McGill University, Montreal, Canada H3A 2T7. Email: ngo.long@mcgill.ca.

${ }^{\ddagger}$ INRA-LAMETA, 2 Place Viala, 34060 cedex 1. Montpellier. France. Email: tidball@supagro.inra.fr. 


\section{Introduction}

This article gets back to Rawls' just savings principle (Rawls, 1971, 1999) and to its link with the recent mixed Bentham-Rawls (MBR) intertemporal choice criterion (Long 2006, Alvarez-Cuadrado \& Long, 2009, Tol, 2013). In a standard dynamic model, we establish general properties of the control path that is deemed optimal according to the MBR criterion; those properties turns out to be, to some extent, consistent with some intuitions about intergenerationnal justice expressed via Rawls' just savings principle.

The general challenge is to think about what we owe to future generations ${ }^{1}$. One possible answer, the just savings principle suggested by Rawls and various scholars working in this field, derives from the social contract approach initiated by Grotius, Hobbes, Locke, Rousseau and Kant. It can be described as the saving rule that an impartial "observer" would deem fair. It advocates a two-phase logic (Gosseries, 2001, Wall, 2003). During a first phase, each generation must save in order to transfer to the next generation more than what it has inherited from the previous generations; the purpose of the accumulation phase is to build up economic conditions so that at least basic freedoms are in place and minimal stability to just institutions can be ensured ${ }^{2}$. Then follows a cruise phase where the principle of equality that prevails recalls the egalitarian-maximin logic, but it is subordinate to the need for an initial take-off from the condition of underdevelopment $^{3}$. Qualitatively, the picture is clear; but when it comes to more operational details, the just saving principle has proven somewhat elusive ${ }^{4}$. Its implications are far too vague and require further precisions. Unfortunately, Rawls did not suggest a precise criterion that would embody his just saving clause $^{5}$.

\footnotetext{
${ }^{1}$ Since the famous UN Brundtland Report (1987), "Our Common Future", and its echo in the political sphere, it is a question that is experiencing a heightened interest; but philosophers - and also economists - have thought about it for a long time.

${ }^{2}$ From Rawls' contractuarian perspective, "the just savings principle can be regarded as an understanding between generations to carry their fair share of the burden of realizing and preserving a just society." And he goes on to say "The [just] savings principle represents an interpretation, arrived at in the original position, of the previously accepted natural duty to uphold and to further just institutions. In this case the ethical problem is that of agreeing on a path over time which treats all generations justly during the whole course of a society's history." (pp. 288-289).

3 "Eventually, once just institutions are firmly established and all the basic liberties effectively realized, the net accumulation asked for falls to zero. At this point a society meets its duty of justice by maintaining just institutions and preserving their material base." (Rawls, 1999).

${ }^{4}$ Several intertemporal social choice criteria used by economists have the ability to prescribe this two-phase logic, though they also imply dramatically different saving rates. This is the case for the "distance-to-bliss" criterion (Ramsey, 1928) and the usual discounted utilitarian criterion (Koopmans, 1960).

${ }^{5} \mathrm{He}$ just hoped that certain extremes will be excluded. One such extreme is typically given by the optimal saving path derived from the undiscounted utilitarian criterion that, by construction, generally demands exorbitant sacrifices to the first generations. Indeed, when investment has a positive return, a sacrifice by the current generation often appears worthwhile when it is pitted against the infinite sum of undiscounted advantages it will produce on subsequent generations. Under plausible specifications of the economy it may yield optimal
} 
The MBR criterion has been suggested as a possibility, where the impartial observer would be a 'Dynasty with Concern for the Least Advantaged' (Long, 2006). And indeed, its application in particular contexts abide by a two-phase logic when the initial conditions are too low, that is to say below the modified golden-rule (Long, 2006, Alvarez-Cuadrado \& Long, 2009). But, beyond those contexts, could this dynamic pattern be a robust property of the optimal path according the MBR criterion? And, since this property also characterize the optimal control for the widely used discounted utilitarian criterion, what is the further argument in favour of the MBR criterion as a superior embodiment of the just saving principle?

This note is organized as follows. The next section presents a simple, but general, dynamic economy that encompasses various applications, and it also explains the MBR criterion. Section 3 establishes and discusses general dynamic properties of the MBR exploitation path; and it also shows that inequalities, captured by the difference of utility between the richest and the poorest generations, is generically lower under the MBR criterion than under the discounted utilitarian criterion. Section 4 concludes.

\section{A dynamic framework and the MBR criterion}

Time is continuous and the horizon is infinite. The economy has infinitely many successive generations. Each generation is made of one representative individual who lives for just one instant. Let $c(t)$ be the control variable, or consumption flow, that affects generation $t$, and the stock variable is $x(t)$. This stock evolves according to the differential equation:

$$
\left\{\begin{array}{c}
\frac{d x(t)}{d t} \equiv \dot{x}(t)=f(x(t), c(t)) \\
x(0)=x_{0} \text { given. }
\end{array}\right.
$$

An admissible path $\{c(),. x()$.$\} is a solution to (1) such that x(t) \geq 0$ and $c(t) \geq 0, \forall t$.

When consuming $c(t)$, generation $t$ enjoys a standard of living, $U(t) \equiv$ $U(c(t))$, where $U($.$) is an increasing function { }^{6}$. To any path $c($.$) let \underline{c}=$ $\inf _{t}\{c()$.$\} stand for the lowest consumption level, and let \underline{U}=U(\underline{c})$ be the corresponding standard of living.

This framework can accommodate two standard interpretations: $i$ ) the RamseySolow optimal growth model, that is obtained as a particular case when $x$ is the capital stock and the dynamics are $\dot{x}=f(x, c)=F(x)-c-\delta x$, where $F($. is a production function such that $F(x) \geq 0, F^{\prime}(x) \geq 0, F^{\prime \prime}(x)<0$, and $\delta>0$ is the rate of depreciation, $i i$ ) the basic renewable resource model, when $x$ is a natural resource that evolves according to the equation $\dot{x}=f(x, c)=G(x)-c$, where $G($.$) is a concave function that reaches a maximum at some x^{M}$, called the maximum sustainable yield.

\footnotetext{
savings amounting to more than 60 percent of gross national product for the first generations.

${ }^{6}$ Well-being experienced by generation $t$ is only but one interpretation that could be given to function $U($.$) .$
} 
To any admissible path, the $M B R$ criterion associates the following value:

$$
W^{m b r}(c(.)) \equiv \theta \underline{U}+(1-\theta) \int_{t=0}^{\infty} e^{-r t} U(c(t)) d t, \quad 0<\theta<1 .
$$

$W^{m b r}$ is a weighted average of the maximin criterion and the usual discounted utilitarian criterion. It can be seen as a procedural compromise between the concern for the worse-off (the larger $\theta$, the stronger this concern) and the concern for all generations with a discount for the position on the temporal axis.

Some properties of the MBR criterion are exposed and discussed at length in Alvarez-Cuadrado \& Long (2009). From a deontologic point of view, let us recall briefly that $W^{m b r}$ meets the following requirements: completeness, strong Pareto, non-dictatorship of the future and non-dictatorship of the present ${ }^{7}$. These properties are important, but we do not dwell on here; they are widely discussed elsewhere (Alvarez-Cuadrado \& Long, 2009), and in any case it is a consequentialist point of view that interests us in this article. To be more precise, the object of interest in the present paper is not the expression (2) itself; rather we shall focus on the properties of the solution to the $M B R$ problem, i.e. the trajectory $\{c(),. x()$.$\} that maximizes (2) subject to (1).$

\section{Properties of optimal trajectories under the MBR criterion}

The economic framework presented above features a minimal structure. Yet, even this basic structure already implies the following property on the endogenous path induced by the MBR criterion.

Theorem 1 (Monotonicity) Let the pair $\left\{c^{m b r}(),. x^{m b r}().\right\}$ be a solution to the $M B R$ problem. Assume that $c^{m b r}($.$) is not constant and x^{m b r}($.$) is unique. Then$ $x^{m b r}($.$) is monotonic for t \in[0,+\infty[$.

Proof. Appendix A.

Note that Theorem 1 does not rest on demanding assumptions on the fundamentals of the economy given by functions $f(.,$.$) and U($.$) . Regarding f(.,$.$) ,$ neither differentiability, nor continuity, nor Lipschitzian assumptions are really necessary. Those kinds of assumptions help to guarantee the existence a solution to the differential equation (1), but they are sufficient and not necessary. We don't need either any concavity assumptions on $U($.$) or any transversality$ conditions. Such conditions are helpful to guarantee that candidate paths are indeed optimal, but they are only sufficient, hence too strong. Theorem 1 sheds

\footnotetext{
${ }^{7}$ An intertemporal social function is complete if it can rank any admissible paths. It satisfies Strong Pareto if it is increasing in any $U_{t} \equiv U(c(t))$. It is a dictatorship of the present when its ranking is not sensitive to the utility of generations located after some date $T$. It is a dictatorship of the future when its ranking is affected only by the utility of generations that are infinitely distant (see Chichilnisky, 1996).
} 
light more directly on the existence of a possible structure on endogenous variables whereby the optimal stock $x$ cannot be cyclical, or increasing and then decreasing and vice versa. Actually, this theorem is a generalisation of Hartl's result (1987) about the monotonicity of the state trajectories in autonomous control problems ${ }^{8}$.

Nevertheless, sufficient conditions can be identified to ensure the MBR problem is strictly concave and therefore its solution is unique. For the sake of completeness, they are given in Theorem 2 below.

Theorem 2 Let $\left(c^{m b r}(),. x^{m b r}(),. \underline{c}^{m b r}\right)$ be a candidate optimal solution, with the associated time path of shadow prices $\left(\psi^{m b r}(),. \lambda^{m b r}().\right)$. Assume that all the necessary conditions are satisfied. Assume that the following transversality conditions are satisfied:

$$
\lim _{t \rightarrow \infty} \psi^{m b r}(t) x^{m b r}(t)=0
$$

and

$$
\lim _{t \rightarrow \infty} \psi^{m b r}(t) \geq 0 .
$$

Consider any alternative feasible plan $\left(c^{\#}(),. x^{\#}(),. \underline{c}^{\#}\right)$. For any $(c, x, \underline{c})$, we define the following Lagrangian using the shadow prices of the candidate optimal path:

$$
\begin{aligned}
L\left(c, x, \underline{c}, \psi^{m b r}, \lambda^{m b r}, t\right) \equiv & e^{-r t}[r \theta U(\underline{c})+(1-\theta) U(c)] \\
& +\psi^{m b r} f(x, c)+\lambda^{m b r}(c-\underline{c}) .
\end{aligned}
$$

Let $V^{m b r}$ and $V^{\#}$ be the payoffs obtained by carrying the plans $\left(c^{m b r}(),. x^{m b r}(),. \underline{c}^{m b r}\right)$ and $\left(c^{\#}(),. x^{\#}(),. \underline{c}^{\#}\right)$ respectively, i.e.

$$
\begin{aligned}
V^{m b r} & =\int_{0}^{\infty} e^{-r t}\left[r \theta U\left(\underline{c}^{m b r}\right)+(1-\theta) U\left(c^{m b r}(t)\right)\right] d t, \\
V^{\#} & =\int_{0}^{\infty} e^{-r t}\left[r \theta U\left(\underline{c}^{\#}\right)+(1-\theta) U\left(c^{\#}(t)\right)\right] d t .
\end{aligned}
$$

Assume that $L$ is concave in $(c, x, \underline{c})$. Then $V^{m b r} \geq V^{\#}$. In the case where $L$ is strictly concave in $(c, x, \underline{c})$, then the optimal solution is unique.

Proof. Appendix B.

Added to the general necessary conditions already given in Alvarez-Cuadrado \& Long (2009) (see also Appendix B of the present paper), the above theorem about sufficient conditions completes the "user kit" of the MBR criterion.

\footnotetext{
${ }^{8}$ Hartl (1987) deals with the discounted utilitarian criterion that, strictly speaking, is a particular case of expression (2) only when $\theta=0$, a value that is ruled out in principle. However this value is forbidden simply to ensure that MBR escapes the dictatorship of the present. But nothing in the proof of Theorem 1 is compromised when $\theta=0$. The proof just becomes simpler.
} 
Next, let us assume in this dynamic framework that $x$ is a productive asset, in the following sense:

Assumption 1 For any pair of points in time $\left(t_{a}, t_{b}\right)$, where $t_{a}<t_{b}$, and any non-negative initial stock level $a$, let $c^{*}($.$) be an admissible trajectory in the time$ interval $\left[t_{a}, t_{b}\right]$, i.e.

$$
\left\{\begin{array}{c}
\dot{x}(t)=f\left(x(t), c^{*}(t)\right), \forall t \in\left[t_{a}, t_{b}\right] \\
x\left(t_{a}\right)=a, x(t) \geq 0, \forall t \in\left[t_{a}, t_{b}\right]
\end{array}\right.
$$

and let $b$ be the resulting stock size at time $t_{b}$,

$$
b \equiv x\left(t_{a}\right)+\int_{t_{a}}^{t_{b}} f\left(x(t), c^{*}(t)\right) d t
$$

Then, for any $\varepsilon>0$, there exists a feasible path $c_{\varepsilon}($.$) in the time interval \left[t_{a}, t_{b}\right]$ such that

$$
c_{\varepsilon}(t) \geq c^{*}(t) \text { for all } t \in\left[t_{a}, t_{b}\right]
$$

and

$$
\left\{\begin{array}{c}
\dot{x}(t)=f\left(x(t), c_{\varepsilon}(t)\right), \forall t \in\left[t_{a}, t_{b}\right], \\
x\left(t_{a}\right)=a+\varepsilon, \quad x\left(t_{b}\right)=b, \quad x(t) \geq 0, \forall t \in\left[t_{a}, t_{b}\right] .
\end{array}\right.
$$

Essentially, this assumption states that if the initial stock gets larger, at least as much consumption as before becomes feasible over an interval, even if at the end of this interval the final stock is unchanged. Under this assumption, we can give some information about the occurence of the poorest generations over the time line.

Theorem 3 Let the pair $\left\{c^{m b r}(),. x^{m b r}().\right\}$ be a solution to the MBR problem. Assume that $c^{m b r}($.$) is not constant and x^{m b r}($.$) is unique. If the stock x$ is a productive asset (Assumption 1) the following properties hold:

1. when $x^{m b r}($.$) is non-constant and weakly-increasing over time, then the$ poorest generations cannot be at the end of the sequence,

2. when $x^{m b r}($.$) is non-constant, and weakly-decreasing over time, then the$ poorest generations cannot be at the beginning of the sequence.

\section{Proof. Appendix C.}

In the perspective of appraising the ability of the MBR path to capture the two-phase logic of the just saving principle, it is the first item in the above theorem, where the stock of the ressource is weakly-increasing, that is relevant. A pattern where the stock increases over time opens the possibility for future generations to enjoy higher levels of consumption. But, a priori, a trajectory where the lowest levels of consumption are in the far future cannot be excluded either, for it is admissible. However, Theorem 3 establishes that this possiblity does not characterize a solution to the MBR problem.

The next results are helpful for comparing the MBR path with the discounted utilitarian (DU) path. 
Corollary 1 Let $\left\{c^{m b r}(),. x^{m b r}().\right\}$ be the unique solution starting from some $x_{0}$. Suppose $x^{m b r}($.$) is non-constant and weakly increasing. Then there exists a$ finite time $T$ such that after time $T$ the solution $\left(x^{m b r}(),. c^{m b r}().\right)$ is the solution of the discounted utilitarian program

$$
\max _{c} \int_{T}^{\infty} u(c) e^{-r t} d t
$$

s.t. $\dot{x}=f(x, c), x(T)=x_{T}^{m b r}$, with $x \geq 0$ and $c \geq 0$. In particular $c^{m b r}(t)>$ $\underline{c}^{m b r}$ for all $t \geq T$.

Proof. This result follows from Claim 1 of Theorem 3. The complete proof is in Appendix D.

The above result is a generalization of Proposition 3, item ( $i i)$, established in Alvarez-Cuadrado \& Long (2009) for a specific renewable resource model. It is the information provided by Theorem 3 that makes this generalization possible.

Theorem 4 Under the assumptions of Corollary 1, if $f(x . c)$ is concave in $(x, c)$ and $u(c)$ is concave then after time $T$ the time path $c^{m b r}($.$) is weakly increasing$ provided that $f_{c}<0$ and $f_{x c} \geq 0$.

Proof. Appendix E.

The above theorem is not only indicative of the behavior of the optimal consumption after some date $T$. It will also prove useful to establish that the upper level of consumption under the MBR criterion is achieved at infinity, i.e. $\sup _{t}\left\{c^{m b r}().\right\}=\lim _{t \rightarrow \infty} c^{m b r}(t)$, and to compare the MBR path with the discounted utilitarian path.

In view of this, consider now the following definition.

Definition 1 Let

$$
I(c(.))=\sup _{t}\{c(.)\}-\inf _{t}\{c(.)\}=\bar{c}-\underline{c}
$$

be the size of consumptions distribution in trajectory $c($.$) .$

One may expect that inequality, as measured by $I(c()$.$) , is lower under the$ MBR criterion than under the discounted utilitarian criterion. This conjecture has to be ascertained. Clearly $\underline{c}^{m b r} \geq \underline{c}^{d u}$, by construction. But what about $\bar{c}^{m b r}$ and $\bar{c}^{d u}$ ?

Theorem 5 Let the assumptions underlying Corollary 1 and Theorem 4 jointly hold. Let $c^{d u}($.$) be the solution to the discounted utilitarian program. Suppose$ $x^{m b r}($.$) is weakly increasing. Then$

$$
I\left(c^{m b r}(.)\right) \leq I\left(c^{d u}(.)\right),
$$

i.e. inequality among generations, as captured by $I(c()$.$) , is lower under the$ $M B R$ criterion than under the discounted utilitarian criterion. 
Proof. Appendix F.

Admittedly, $I(c()$.$) is coarse indicator of inequality. But it is relevant here,$ when comparing $c^{m b r}($.$) and c^{d u}($.$) , because, when x^{m b r}($.$) is weakly increasing,$ both trajectories share the same upper-level of consumption $\bar{c}$, as can be deduced from Corollary 1. Therefore, inequalities between generations that take place in the intervals $\bar{c}-\underline{c}^{m b r}$ and $\bar{c}-\underline{c}^{d u}$ could be very different, but at least the poorest are less far from the richest under the MBR scenario. The MBR path outperforms the DU path in the perspective to capture the rawlsian idea that inequalities can be justified when they benefit the most disadvantaged people.

\section{Conclusion}

This paper provides general theorems in respect of the control that maximizes the MBR intertemporal choice function. The main results are as follows: $i$ ) the state variable is shown to be monotonic under rather weak conditions, ii) we establish sufficient concavity conditions for a candidate trajectory to be optimal and unique, iii) and we prove that inequality among generations, captured by the gap between the poorest and the richest generations, is lower when optimization is performed under the MBR criterion rather than under the discounted utilitarian criterion.

Those results are helpful to give a content to Rawls' (1971) just saving principle. The vagueness of this principle, in its implications, allows multiple interpretations. Several intertemporal social choice criteria, familiar to economists, could be contenders as incarnations of this principle. How are we to choose among them? According to Rawls, our final conception of justice should establish what he calls a "reflective equilibrium" - an acceptable balance between, on the one hand, deontological principles of justice and, on the other hand the consequences of applying those general principles to specific cases.

Following this methodology, we could then scrutinize various social welfare criteria and check, from a consequentialist point of view, their compliance to at least two aspects of the just saving principle: $i$ ) the necessity of a take-off phase if the initial conditions are two low, $i i$ ) that inequalities, if any, should benefit the less advantaged generations.

It is well know that the maximin criterion, sometimes erroneously attributed to Rawls, violates condition $i$ ). And Rawls also rejected the undiscounted utilitarian criterion. This criterion can be consistent with point $i$ ) but, by construction, it generally demands exorbitant sacrifices to the first generations, therefore it does not comply with point $i i$ ). From this perspective, it may be argued that the practice of discounting future advantages, prescribed under Koopmans' logic (1960), is not so unfair after all. This is so because productive investment features an in-built bia s in favour of the future that can be redressed by granting more importance to earlier generations. This is a standard argument, sometimes supported, sometimes challenged by economists who are working on climate change (see for instance the synthesis given by Dasgupta, 2008, or the critical assessment of Roemer, 2011, in particular Section 3 of his paper, or Section 5.1 
in Asheim, 2010). Although it appears unfair from a deontological perspective (a dictatorship of the present in the words of Chichilnisky, 1996), the discounted utilitarian criterion might be a not so bad candidate to embody the just savings principle. But, clearly, our comparison of the optimal trajectories under the DU and the MBR criterion (Theorem 5) does not support this conclusion.

\section{Aknowledgements}

We thank Geir Asheim, Luc Doyen and Michel De Lara for helpful comments.

\section{References}

[1] Alvarez-Cuadrado F. and Ngo Van Long (2009), "A Mixed Bentham-Rawls Criterion for Intergenerational Equity: Theory and Implications", Journal of Environmental Economics and Management, 58, 154-168.

[2] Asheim G. B (2010), "Intergenerational Equity", Annual Review of Economics, 2, 197-122.

[3] Chichilnisky G. (1996), "An Axiomatic Approach to Sustainable Development", Social Choice and Welfare, 13, 231-57.

[4] Dasgupta P. (2008), "Discounting climate change," Journal of Risk and Uncertainty, 37(2), 141-169.

[5] Dasgupta P. (2005), "Three Conceptions of Intergenerational Justice " in: H. Lillehammer and D.H. Mellor, eds (2005), Ramsey's Legacy (Oxford: Clarendon Press)).

[6] Gosseries A. P. (2001), "What Do We Owe the Next Generation(s)", 35 Loyola of Los Angeles Law Review 293.

[7] Koopmans T., (1960), "Stationary Ordinal Utility and Impatience", Econometrica 28, 287-309.

[8] Hartl, Richard F. (1987), "A Simple Proof of the Monotonicity of the State Trajectories in Autonomous Control Problems", Journal of Economic Theory, 41, issue 1, p. 211-215

[9] Long, N. V. (1979), "Two Theorems on Generalized Diminishing Returns and their Applications to Economic Analysis," Economic Record, 55, 58-63.

[10] Long, N. V. (2007), "Toward a Theory of a Just Savings Principle', in J. Roemer and K. Suzumura (eds.) Intergenerational Equity and Sustainability, Palgrave, London, 2007, 291-319. 
[11] Nicolescu, C. and Lars-Erik Persson, L.-E. (2006), "Convex Functions and their Applications : A Contemporary Approach", coll. « Ouvrages de mathématiques de la Société mathématique du Canada », vol. 23.

[12] Ramsey F. (1928), "A Mathematical Theory of Savings", Economic Journal, 38, 543-559.

[13] Rawls, J. (1999) A Theory of Justice (Revised Edition), Cambridge, Mass:The Belknap Press of the Harvard University Press.

[14] Roemer J. (2011), "The Ethics of Intertemporal Distribution in a Warming Planet", Environmental $\& 3$ Resource Economics, 48(3), 363-390.

[15] Tol R. S.J. (2013), "Climate policy with Bentham-Rawls preferences", Economics Letters, 118(3), 424-428.

[16] Wall, S. (2003), "Just Savings and the Difference Principle." Philosophical Studies 116.1, 79-102.

\section{A Proof of Theorem 1}

The proof is by contradiction. Assume that $x^{m b r}($.$) is not monotonic. Then,$ there exists a date $\tau \in[0,+\infty[$ and a strictly positive number $\theta$ such that:

$$
x^{m b r}(\tau)=x^{m b r}(\tau+\theta),
$$

with $x^{m b r}($.$) not constant on the interval [\tau, \tau+\theta]$.

Now define a new admissible path $\{\widetilde{c}(),. \widetilde{x}()$.$\} with control and stable vari-$ ables constructed as follows:

$$
\left\{\begin{array}{c}
\widetilde{c}(t)=c^{m b r}(t), \quad \widetilde{x}(t)=x^{m b r}(t), \quad \forall t \in[0, \tau], \\
\left.\widetilde{c}(t)=c^{m b r}(t+\theta), \quad \widetilde{x}(t)=x^{m b r}(t+\theta), \quad \forall t \in\right] \tau,+\infty[.
\end{array}\right.
$$

By construction the pair $\{\widetilde{c}(),. \widetilde{x}()$.$\} is admissible. And \widetilde{c}($.$) can also be made$ different from $c^{m b r}($.$\left.) over the interval \right] \tau,+\infty[$. Indeed, if on the contrary $\widetilde{c}(t)=$ $\left.c^{m b r}(t+\theta)=c^{m b r}(t), \forall t \in\right] \tau,+\infty\left[\right.$, then by definition $c^{m b r}(t)$ is periodic and not constant by assumption - on $] \tau,+\infty[$. In such a case one can simply choose two alternative numbers $\tau^{\prime}, \theta^{\prime}$ such that $\left[\tau^{\prime}, \tau^{\prime}+\theta^{\prime}\right] \sqsubset[\tau, \tau+\theta]$ and construct the above alternative path $\{\widetilde{c}(),. \widetilde{x}()$.$\} using date \tau^{\prime}$ and $\theta^{\prime}$ instead of $\tau$ and $\theta$. Clearly $\{\widetilde{c}(),. \widetilde{x}()$.$\} is different from \left\{c^{m b r}(),. x^{m b r}().\right\}$ on the interval ]$\tau^{\prime},+\infty[$. To simplify, let us just consider that $\{\widetilde{c}(),. \widetilde{x}()$.$\} is different from$ $\left\{c^{m b r}(),. x^{m b r}().\right\}$ on the interval $] \tau,+\infty[$.

Also, by construction,

$$
\underline{c}^{m b r}=\inf _{t}\left\{c^{m b r}(.)\right\} \leq \underline{\widetilde{c}}=\inf _{t}\{\widetilde{c}(.)\}
$$


or equivalently, using obvious notations

$$
\underline{U}^{m b r} \leq \underline{\widetilde{U}} .
$$

By definition $c^{m b r}($.$) is the unique optimal solution, and necessarily:$

$$
W^{m b r}\left(c^{m b r}(.)\right)>W^{m b r}(\widetilde{c}(.)),
$$

or,

$$
\theta \underline{U}^{m b r}+(1-\theta) \int_{t=0}^{\infty} e^{-r t} U\left(c^{m b r}(t)\right) d t>\theta \underline{\widetilde{U}}+(1-\theta) \int_{t=0}^{\infty} e^{-r t} U(\widetilde{c}(t)) d t .
$$

Hence,

$$
\begin{array}{ll}
\int_{t=0}^{\infty} e^{-r t} U\left(c^{m b r}(t)\right) d t & >\int_{t=0}^{\infty} e^{-r t} U(\widetilde{c}(t)) d t, \\
\int_{t=\tau}^{\infty} e^{-r t} U\left(c^{m b r}(t)\right) d t>\int_{t=\tau}^{\infty} e^{-r t} U(\widetilde{c}(t)) d t, & \\
\int_{t=\tau}^{\infty} e^{-r t} U\left(c^{m b r}(t)\right) d t>\int_{t=\tau}^{\infty} e^{-r t} U\left(c^{m b r}(t+\theta)\right) d t,
\end{array}
$$

where the second line obtains because the two controls coincide until date $\tau$, and the last line makes use of (5). Notice that:

$$
\int_{t=\tau}^{\infty} e^{-r t} U\left(c^{m b r}(t+\theta)\right) d t=e^{r \theta} \int_{t=\tau+\theta}^{\infty} e^{-r t} U\left(c^{m b r}(t)\right) d t .
$$

With this expression, inequality (6) can be written:

$$
\int_{t=\tau}^{\infty} e^{-r t} U\left(c^{m b r}(t)\right) d t>e^{r \theta} \int_{t=\tau+\theta}^{\infty} e^{-r t} U\left(c^{m b r}(t)\right) d t,
$$

consequently:

$$
\int_{t=\tau}^{\tau+\theta} e^{-r t} U\left(c^{m b r}(t)\right) d t>\left(e^{r \theta}-1\right) \int_{t=\tau+\theta}^{\infty} e^{-r t} U\left(c^{m b r}(t)\right) d t .
$$

Next define a new admissible path $\{\bar{c}(),. \bar{x}()$.$\} with control and stable vari-$ able now constructed as follows:

$$
\left\{\begin{array}{c}
\bar{c}(t)=c^{m b r}(t), \bar{x}(t)=x^{m b r}(t), \quad \forall t \in[0, \tau+\theta], \\
\left.\bar{c}(t)=c^{m b r}(t-\theta), \quad \bar{x}(t)=x^{m b r}(t-\theta), \quad \forall t \in\right] \tau+\theta,+\infty[.
\end{array}\right.
$$


Again, by construction the pair $\{\bar{c}(),. \bar{x}()$.$\} is admissible and different from$ $\left\{c^{m b r}(),. x^{m b r}().\right\}$. Also by construction,

$$
\underline{c}^{m b r}=\inf _{t}\left\{c^{m b r}(.)\right\}=\underline{\bar{c}}=\inf _{t}\{\bar{c}(.)\}
$$

or

$$
\underline{U}^{m b r}=\underline{\bar{U}} .
$$

We now compare the value of $W^{m b r}($.$) for \{\bar{c}(),. \bar{x}()$.$\} and \left\{c^{m b r}(),. x^{m b r}().\right\}$. Using definition (8), equality (9) and inequality (7):

$$
\begin{aligned}
W^{m b r}(\bar{c}(.))-W^{m b r}\left(c^{m b r}(.)\right) & =\int_{t=0}^{\infty} e^{-r t} U(\bar{c}(t)) d t-\int_{t=0}^{\infty} e^{-r t} U\left(c^{m b r}(t)\right) d t \\
& =\int_{t=\tau+\theta}^{\infty} e^{-r t} U\left(c^{m b r}(t-\theta)\right) d t-\int_{t=\tau+\theta}^{\infty} e^{-r t} U\left(c^{m b r}(t)\right) d t \\
& =e^{-r \theta} \int_{t=\tau}^{\infty} e^{-r t} U\left(c^{m b r}(t)\right) d t-\int_{t=\tau+\theta}^{\infty} e^{-r t} U\left(c^{m b r}(t)\right) d t \\
& =e^{-r \theta}\left[\int_{t=\tau}^{\tau+\theta} e^{-r t} U\left(c^{m b r}(t)\right) d t-\left(e^{r \theta}-1\right) \int_{t=\tau+\theta}^{\infty} e^{-r t} U\left(c^{m b r}(t)\right) d t\right]>0,
\end{aligned}
$$

a contradiction.

\section{B Necessary conditions, sufficient conditions and uniqueness}

\section{B.1 Necessary conditions}

First, recall the following identity

$$
1=\int_{0}^{\infty} r e^{-r t} d t
$$

Thus, a solution to the MBR problem is a triple $\left(c^{m b r}(),. x^{m b r}(),. \underline{c}^{m b r}\right)$ that maximizes

$$
\int_{0}^{\infty} e^{-r t}[r \theta U(\underline{c})+(1-\theta) U(c(t))] d t
$$

subject to

$$
\begin{gathered}
\dot{x}=f(x(t), c(t)), \\
c(t)-\underline{c} \geq 0 .
\end{gathered}
$$


Following the approach of Hestenes", we treat $\underline{c}$ as a "control parameter", i.e. a variable that,once chosen, remains constant over the time horizon $[0, \infty[$. We define the Lagragian:

$$
L=e^{-r t}[r \theta U(\underline{c})+(1-\theta) U(c(t))]+\psi(t) f(x(t), c(t))+\lambda(t)(c(t)-\underline{c})
$$

The necessary conditions are:

$$
\begin{gathered}
\frac{\partial L}{\partial c}=0 \\
\dot{\psi}=-\frac{\partial L}{\partial x}, \\
\dot{x}=\frac{\partial L}{\partial \psi}, \\
\lambda(t) \geq 0, c(t)-\underline{c} \geq 0, \lambda(t)(c(t)-\underline{c})=0, \\
\frac{\partial}{\partial \underline{c}} \int_{0}^{\infty} e^{-r t}[r \theta U(\underline{c})+(1-\theta) U(c(t))] d t+\frac{\partial}{\partial \underline{c}} \int_{0}^{\infty} \lambda(t)(c(t)-\underline{c}) d t=0 .
\end{gathered}
$$

The latter condition reduces to

$$
\theta U^{\prime}(\underline{c})-\int_{0}^{\infty} \lambda(t) d t=0
$$

Adn the transversality conditions are:

$$
\begin{aligned}
\lim _{t \rightarrow \infty} \psi(t) & \geq 0, \\
\lim _{t \rightarrow \infty} \psi(t) x(t) & =0 .
\end{aligned}
$$

\section{B.2 Sufficient conditions and uniqueness}

Our proof is similar to that of Takayama (1986).

For simplicity, we use the following notations

$$
L^{m b r}=L\left(c^{m b r}, x^{m b r}, \underline{c}^{m b r}, \psi^{m b r}, \lambda^{m b r}, t\right),
$$

and

$$
L^{\#}=L\left(c^{\#}, x^{\#}, \underline{c}^{\#}, \psi^{m b r}, \lambda^{m b r}, t\right), \quad\left(\text { note: } \psi^{m b r}, \lambda^{m b r}\right. \text { are not typos here), }
$$

where the "mbr" over the multipliers indicates that we use the same path $\left(\psi^{m b r}(),. \lambda^{m b r}().\right)$ for both $L^{m b r}$ and $L^{\#}$.

Since $\lambda^{m b r}\left[c^{m b r}-\underline{c}^{m b r}\right]=0$,

$$
V^{m b r}=\int_{0}^{\infty}\left[L^{m b r}-\psi^{m b r} \dot{x}^{m b r}\right] d t
$$

\footnotetext{
${ }^{9}$ See, for example, Takayama, A. (1986), Mathematical Economics, second edition, Cambridge University Press, Cambridge and New York
} 
Now, since $\lambda^{m b r} \geq 0$ and since, by feasibility, $c^{\#}-\underline{c}^{\#} \geq 0$, we have $\lambda^{m b r}\left(c^{\#}-\underline{c}^{\#}\right) \geq$ 0 , hence

$$
V^{\#}=\int_{0}^{\infty}\left[L^{\#}-\psi^{m b r} \dot{x}^{\#}-\lambda^{m b r}\left(c^{\#}-\underline{c}^{\#}\right)\right] d t \leq \int_{0}^{\infty}\left[L^{\#}-\psi^{m b r} \dot{x}^{\#}\right] d t
$$

Then

$$
\begin{aligned}
V^{m b r}-V^{\#} \geq & -\int_{0}^{\infty}\left[\psi^{m b r} \dot{x}^{m b r}-\psi^{m b r} \dot{x}^{\#}\right] d t \\
& +\int_{0}^{\infty}\left[L^{m b r}-L^{\#}\right] d t
\end{aligned}
$$

Now, under the assumption that $L$ is concave

$$
L^{m b r}-L^{\#} \geq\left(x^{m b r}-x^{\#}\right) \frac{\partial L^{m b r}}{\partial x}+\left(c^{m b r}-c^{\#}\right) \frac{\partial L^{m b r}}{\partial c}+\left(\underline{c}^{m b r}-\underline{c}^{\#}\right) \frac{\partial L^{m b r}}{\partial \underline{c}}
$$

with strict inequality if $L$ is strictly concave.

Now from the necessary conditions $\frac{\partial L^{m b r}}{\partial c}=0, \frac{\partial L^{m b r}}{\partial x}=-\dot{\psi}^{m b r}$. Then

$$
L^{m b r}-L^{\#} \geq-\dot{\psi}^{m b r}\left(x^{m b r}-x^{\#}\right)+\left(\underline{c}^{m b r}-\underline{c}^{\#}\right) \frac{\partial L^{m b r}}{\partial \underline{c}}
$$

Therefore

$$
\begin{gathered}
V^{m b r}-V^{\#} \geq-\int_{0}^{\infty}\left[\dot{\psi}^{m b r}\left(x^{m b r}-x^{\#}\right)+\psi^{m b r} \dot{x}^{m b r}-\psi^{m b r} \dot{x}^{\#}\right] d t+ \\
+\left(\underline{c}^{m b r}-\underline{c}^{\#}\right) \int_{0}^{\infty}\left[\frac{\partial L^{m b r}}{\partial \underline{c}}\right] d t
\end{gathered}
$$

Since $\int_{0}^{\infty}\left[\frac{\partial L^{m b r}}{\partial \underline{c}}\right] d t=0$ by condition (10), we obtain

$V^{m b r}-V^{\#} \geq-\lim _{t \rightarrow \infty}\left[\psi^{m b r}(t) x^{m b r}(t)-\psi^{m b r}(0) x^{m b r}(0)\right]+\lim _{t \rightarrow \infty}\left[\psi^{m b r}(t) x^{\#}(t)-\psi^{m b r}(0) x^{\#}(0)\right]$

Using the fixed initial condition, $x_{0}^{m b r}=x_{0}^{\#}=x_{0}$, the above inequality becomes

$$
V^{m b r}-V^{\#} \geq \lim _{t \rightarrow \infty} \psi^{m b r}(t)\left[x^{\#}(t)-x^{m b r}(t)\right]
$$

which is positive as can be deduced from the transversality conditions (11) and (12) and because $x^{\#}(t) \geq 0$. With a strictly concave $L$, we obtain uniqueness.

\section{Proof of Theorem 3}

Claim 1. Recall that, if $c^{m b r}($.$) and x^{m b r}($.$) are a solution to the MBR problem$ where $c^{m b r}($.$) is not constant and x^{m b r}($.$) is unique, then x^{m b r}($.$) is monotonic$ (by Theorem 1). 
In order to prove Claim 1, assume on the contrary that $x^{m b r}($.$) is non-$ constant and weakly increasing over time but the poorest generation(s) is/are at the end of the sequence. Let $\bar{t}>0$ be the earliest date at which the lowest level of consumption is achieved. That is,

$$
\bar{t} \equiv \inf _{t}\left\{t: c^{m b r}\left(t^{\prime}\right)=\underline{c}^{m b r}, \forall t^{\prime} \geq t\right\}
$$

Thus, after $\bar{t}$, the consumption path is constant.

Then, there exists a number $d>0$ such that $c^{m b r}(\bar{t}-d)>c^{m b r}(\bar{t})=\underline{c}^{m b r}$ and $c^{m b r}\left(\bar{t}-d^{\prime}\right)>\underline{c}^{m b r}$ for all $d^{\prime} \in(0, d)$.

At time $\bar{t}-d$, the stock is $x^{m b r}(\bar{t}-d) \leq x^{m b r}(\bar{t})$. Since the stock $x^{m b r}(\bar{t}-d)$ can sustain a stream of consumption with an initial phase of length $d$ with $c>c^{m b r}(\bar{t})$ followed by a phase of constant consumption $\underline{c}^{m b r}$, it follows from Assumption 1 that starting from time $\bar{t}$ with stock level $x^{m b r}(\bar{t}) \geq x^{m b r}(\bar{t}-d)$, it is possible to sustain a stream of consumption $c^{* *}$ with an initial phase $[\bar{t}, \bar{t}+d]$ such that $c^{* *}(t)>c^{m b r}(t)$ and for all $t \in[\bar{t}, \bar{t}+d]$, and $c^{* *}(t)=\underline{c}^{m b r}$ for all $t>\bar{t}+d$.

To summarize, the following alternative sequence $c^{* *}($.$) is admissible:$

$$
\left\{\begin{array}{c}
c^{* *}(t)=c^{m b r}(t), x^{* *}(t)=x^{m b r}(t), \quad \forall t \in[0, \bar{t}[ \\
c^{* *}(t) \geq c^{m b r}(t-d), \quad \forall t \in[\bar{t},+\infty[, \text { with equality for all } t \in[\bar{t}+d,+\infty[
\end{array}\right.
$$

and, by construction, consumptions under the two possibilities are identical except over the interval $[\bar{t}, \bar{t}+d]$ where one has $c^{* *}(t)>c^{m b r}(t)$.

Comparing the value of the MBR criterion under the optimal path and the alternative path, one has:

$$
\begin{aligned}
W^{m b r}\left(c^{m b r}(.)\right)-W^{m b r}\left(c^{* *}(.)\right) & =\int_{\substack{t=0 \\
\bar{t}+d}}^{\infty} e^{-r t} U\left(c^{m b r}(t)\right) d t-\int_{t=0}^{\infty} e^{-r t} U\left(c^{* *}(t)\right) d t, \\
& \leq \int_{t=\bar{t}}^{-r t} \underbrace{\left[U\left(c^{m b r}(t)\right)-U\left(c^{*}(t-d)\right)\right]}_{<0} d t<0,
\end{aligned}
$$

a contradiction.

Claim 2. The proof follows a logic similar to that of Claim 1. Assume on the contrary that $x^{m b r}$ (.) is non-constant and weakly decreasing over time, but the poorest generation(s) is/are at the beginning of the sequence. Suppose there exists an initial interval $[0, \bar{t}]$, with $c^{m b r}(t)=\underline{c}^{m b r}$, and there exists $\delta>0$ such that $c^{m b r}(t)>c^{m b r}(\bar{t}), \forall t \in(\bar{t}, \bar{t}+\delta)$, and $c^{m b r}(t) \geq c^{m b r}(\bar{t})$ for all $t \geq \bar{t}+\delta$.

Since $x^{m b r}(0) \geq x^{m b r}(\bar{t})$, it follows from Assumption 1 that we can construct a time path $c^{* *}(t)$ such that $c^{* *}(t)=c^{m b r}(t+\bar{t})$, for all $t \geq 0$. Comparing the value of the MBR criterion under the optimal path and the alternative path, 
one has:

$$
\begin{aligned}
W^{m b r}\left(c^{m b r}(.)\right)-W^{m b r}\left(c^{* *}(.)\right) & =\int_{t=0}^{\infty} e^{-r t}\left\{U\left(c^{m b r}(t)\right)-\underline{U}\right\} d t-\int_{t=0}^{\infty} e^{-r t}\left\{U\left(c^{* *}(t)\right)-\underline{U}\right\} d t<0 \\
& =\int_{t=\bar{t}}^{\infty} e^{-r t}\left\{U\left(c^{m b r}(t)\right)-\underline{U}\right\} d t-\int_{\tau=0}^{\infty} e^{-r \tau}\left\{U\left(c^{m b r}(\tau+\bar{t})\right)-\underline{U}\right\} d \tau \\
& =\int_{t=\bar{t}}^{\infty} e^{-r t}\left\{U\left(c^{m b r}(t)\right)-\underline{U}\right\} d t-\int_{t=\bar{t}}^{\infty} e^{-r(t-\bar{t})}\left\{U\left(c^{m b r}(t)\right)-\underline{U}\right\} d t<0
\end{aligned}
$$

a contradiction.

\section{Proof of Corollary 1}

Recall the necessary conditions of optimality for the MBR problem given in appendix B. They are:

$$
\begin{aligned}
& \text { i) } \frac{\partial L}{\partial c}=0 \\
& \Leftrightarrow \quad e^{-r t}(1-\theta) U^{\prime}(c(t))+\psi(t) f_{2}(x(t), c(t))+\lambda(t)=0 \\
& \text { ii) } \dot{\psi}=-\frac{\partial L}{\partial x} \text {, } \\
& \Leftrightarrow \quad \dot{\psi}=-\psi(t) f_{1}(x(t), c(t)) \\
& \text { iii) } \dot{x}=\frac{\partial L}{\partial \psi}, \\
& \Leftrightarrow \quad \dot{x}=f(x(t), c(t)) \\
& \text { iv) } \quad \lambda(t) \geq 0, c(t)-\underline{c} \geq 0, \lambda(t)(c(t)-\underline{c})=0 \\
& \text { v) } \frac{\partial}{\partial \underline{c}} \int_{0}^{\infty} e^{-r t}[r \theta U(\underline{c})+(1-\theta) U(c(t))] d t+\frac{\partial}{\partial \underline{c}} \int_{0}^{\infty} \lambda(t)(c(t)-\underline{c}) d t=0
\end{aligned}
$$

The latter condition reduces to

$$
\theta U^{\prime}(\underline{c})-\int_{0}^{\infty} \lambda(t) d t=0
$$

Now, consider that $c(t)>\underline{c}, \forall t \geq T$, (and the stock achieved at date $T$ is $\left.x(T)=x_{T}^{m b r}\right)$. From condition $i v$ ) one can deduce $\lambda(t)=0, \forall t \geq T$, and the necessary conditions boils down to:

$$
\begin{aligned}
e^{-r t}(1-\theta) U^{\prime}(c(t))+\psi(t) f_{2}(x(t), c(t)) & =0 \\
\dot{\psi} & =-\psi(t) f_{1}(x(t), c(t)) \\
\dot{x} & =f(x(t), c(t)) \\
\theta U^{\prime}(\underline{c}) & =\int_{0}^{T} \lambda(t) d t
\end{aligned}
$$


Define the new variable $\sigma(t)=\psi(t) /(1-\theta)$. Then the three first conditions above can be rewritten as:

$$
\begin{aligned}
e^{-r t} U^{\prime}(c(t))+\sigma(t) f_{2}(x(t), c(t)) & =0, \\
\dot{\sigma} & =-\sigma(t) f_{1}(x(t), c(t)), \\
\dot{x} & =f(x(t), c(t)),
\end{aligned}
$$

$\forall t \geq T$. The proof is completed once one observes that these expressions are the necessary conditions associated to the discounted utilitarian program that starts at date $T$ with initial condition $x(T)=x_{T}^{m b r}$, and where the Hamiltonian is:

$$
H=e^{-r t} U(c(t))+\sigma(t) f(x(t), c(t)) .
$$

\section{E Proof of Theorem 4}

From date $T$ the program is utilitarian, and the value function $V(x)$ is concave because $f(x . c)$ is concave in $(x, c)$ and $U(c)$ is concave. ${ }^{10}$ Also, because $V(x)$ is concave, then it is continuous and differentiable almost everywhere (see Nicolescu \& Person, 2006), that is the set of points $x$ where the left hand and the right hand derivatives of $V(x)$, which we can denote by $V_{L}^{\prime}(x)$ and $V_{R}^{\prime}(x)$, are different is at most countable.

Take any two points of time, $t_{1}$ and $t_{2}$, such that $t_{2}>t_{1}$. Then $x\left(t_{2}\right) \geq x\left(t_{1}\right)$ (by assumption). First we analyze the case where $V($.$) is differentiable. And$ after we consider the case where the right hand and left hand derivative of $V($. do not coïncide.

Because $x\left(t_{2}\right) \geq x\left(t_{1}\right)$, then $V^{\prime}\left(x_{2}\right) \leq V^{\prime}\left(x_{1}\right)$ (by concavity of $\left.V().\right)$. We now show that $c\left(t_{2}\right) \geq c\left(t_{1}\right)$. For simplicity of notation, we write $x_{i}$ and $c_{i}$ for $x\left(t_{i}\right)$ and $c\left(t_{i}\right)$, for $i=1,2$.

The HJB equation is

$$
r V(x)=\max _{c}\left[U(c)+V^{\prime}(x) f(x, c)\right] .
$$

Then, the first order condition for the right-hand-side is:

$$
U^{\prime}(c)+V^{\prime}(x) f_{c}(x, c)=0 .
$$

Therefore:

$$
U^{\prime}\left(c_{2}\right)=-f_{c}\left(x_{2}, c_{2}\right) V^{\prime}\left(x_{2}\right)
$$

and

$$
\begin{aligned}
U^{\prime}\left(c_{1}\right) & =-f_{c}\left(x_{1}, c_{1}\right) V^{\prime}\left(x_{1}\right) \\
& =-f_{c}\left(x_{2}, c_{2}\right) V^{\prime}\left(x_{1}\right)+V^{\prime}\left(x_{1}\right)\left[f_{c}\left(x_{2}, c_{2}\right)-f_{c}\left(x_{1}, c_{1}\right)\right] \\
& =-f_{c}\left(x_{2}, c_{2}\right) V^{\prime}\left(x_{1}\right)+V^{\prime}\left(x_{1}\right)\left\{\left[f_{c}\left(x_{2}, c_{2}\right)-f_{c}\left(x_{1}, c_{2}\right)\right]+\left[f_{c}\left(x_{1}, c_{2}\right)-f_{c}\left(x_{1}, c_{1}\right)\right]\right\}
\end{aligned}
$$

${ }^{10}$ See Long (1979) for a proof of the concavity of $V(x)$. 
Then

$$
\begin{gathered}
{\left[U^{\prime}\left(c_{1}\right)-U^{\prime}\left(c_{2}\right)\right]+V^{\prime}\left(x_{1}\right)\left[f_{c}\left(x_{1}, c_{1}\right)-f_{c}\left(x_{1}, c_{2}\right)\right]=} \\
-f_{c}\left(x_{2}, c_{2}\right)\left[V^{\prime}\left(x_{1}\right)-V^{\prime}\left(x_{2}\right)\right]+V^{\prime}\left(x_{1}\right)\left[f_{c}\left(x_{2}, c_{2}\right)-f_{c}\left(x_{1}, c_{2}\right)\right]
\end{gathered}
$$

The right-hand side is positive or zero, because $f_{c}\left(x_{2}, c_{2}\right)-f_{c}\left(x_{1}, c_{2}\right) \geq 0$ (this follows from $\left.f_{x c} \geq 0\right)$. Therefore the left hand side must be positive or zero. This implies that $c_{2} \geq c_{1}$. (Suppose $c_{2}<c_{1}$; then the left hand side would be negative, since the functions $U^{\prime}$ and $f_{c}$ are decreasing in $c$; therefore we would have a contradiction).

In order to complete the proof, let us see what happens if $V$ is not differentiable at $x_{1}$ or at $x_{2}$ ? Then in equation $(15), V^{\prime}(x)$ may correspond to the left hand or the right hand derivative, $V_{L}^{\prime}(x)$ and $V_{R}^{\prime}(x)$. The proof is still valid, because if $x_{2} \geq x_{1}$ then the concavity of $V$ implies

$$
\min \left\{V_{L}^{\prime}\left(x_{1}\right), V_{R}^{\prime}\left(x_{1}\right)\right\} \geq \max \left\{V_{L}^{\prime}\left(x_{2}\right), V_{R}^{\prime}\left(x_{2}\right)\right\}
$$

Then the RHS of (16) is still positive or zero, regardlless of which derivatives we used. Any two paths of $c($.$) that differ from each other at isolated points in$ time are essentially identical, and the resulting path of the state variable is not affected.

\section{F Proof of Theorem 5}

First observe that

$$
\lim _{t \rightarrow \infty} c^{d u}(t)=\bar{c}^{d u},
$$

because, under the assumptions of Theorem 4, consumption is weakly increasing over time. And, as usual, the value of the steady state does not depend on the initial condition.

Observe also that, by virtue of Corollary 1:

$$
\lim _{t \rightarrow \infty} c^{m b r}(t)=\bar{c}^{d u} .
$$

We can also establish that

$$
\lim _{t \rightarrow \infty} c^{m b r}(t)=\bar{c}^{m b r} .
$$

Indeed assume on the contrary that the more advantaged generations occur in finite time at some date $T^{\prime}$. Necessarily this date occurs before the date $T$ at which the MBR trajectory has increasing consumptions (Theorem 4). Then $c^{m b r}\left(T^{\prime}\right)>\lim _{t \rightarrow \infty} c^{m b r}(t)$ and there exists a number $d$ such that:

$$
c^{m b r}(t) \geq \lim _{t \rightarrow \infty} c^{m b r}(t) \quad \forall t \in\left[T^{\prime}, T^{\prime}+d[.\right.
$$


Then consider the alternative consumption trajectory:

$$
\left\{\begin{array}{c}
\widehat{c}(t)=c^{m b r}(t), \forall t \in[0, T[, \\
\widehat{c}(t)=c^{m b r}\left(T^{\prime}+t\right), \forall t \in[T, T+d[, \\
\widehat{c}(t)=c^{m b r}(t), \forall t \in[T+d,+\infty[
\end{array}\right.
$$

This trajectory is admissible and has the same consumptions as the one that maximizes the MBR criterion, except over a finite interval where generations enjoy a higher consumption than under the MBR solution, a contradiction. Therefore we have established $\lim _{t \rightarrow \infty} c^{m b r}(t)=\bar{c}^{m b r}$. To summarize:

$$
\bar{c}^{m b r}=\bar{c}^{d u} .
$$

Since by definition it is also true that $\underline{c}^{m b r} \geq \underline{c}^{d u}$, necessarily:

$$
I\left(c^{m b r}(.)\right)=\bar{c}^{m b r}-\underline{c}^{m b r} \leq I\left(c^{d u}(.)\right)=\bar{c}^{d u}-\underline{c}^{d u} .
$$

QED. 


\section{Documents de Recherche parus en 2013}

DR n²013-01: Estelle MIDLER, Charles FIGUIÈRES, Marc WILLINGER "Choice overload, coordination and inequality: threehurdles to the effectiveness of the compensationmechanism?»

DR n²013-02: Charles FIGUIÈRES, Ngo Van LONGY, Mabel TIDBALL

«The MBR Intertemporal Choice Criterion and Rawls' Just Savings Principle» 
Contact :

Stéphane MUSSARD : mussard@lameta.univ-montp1.fr 


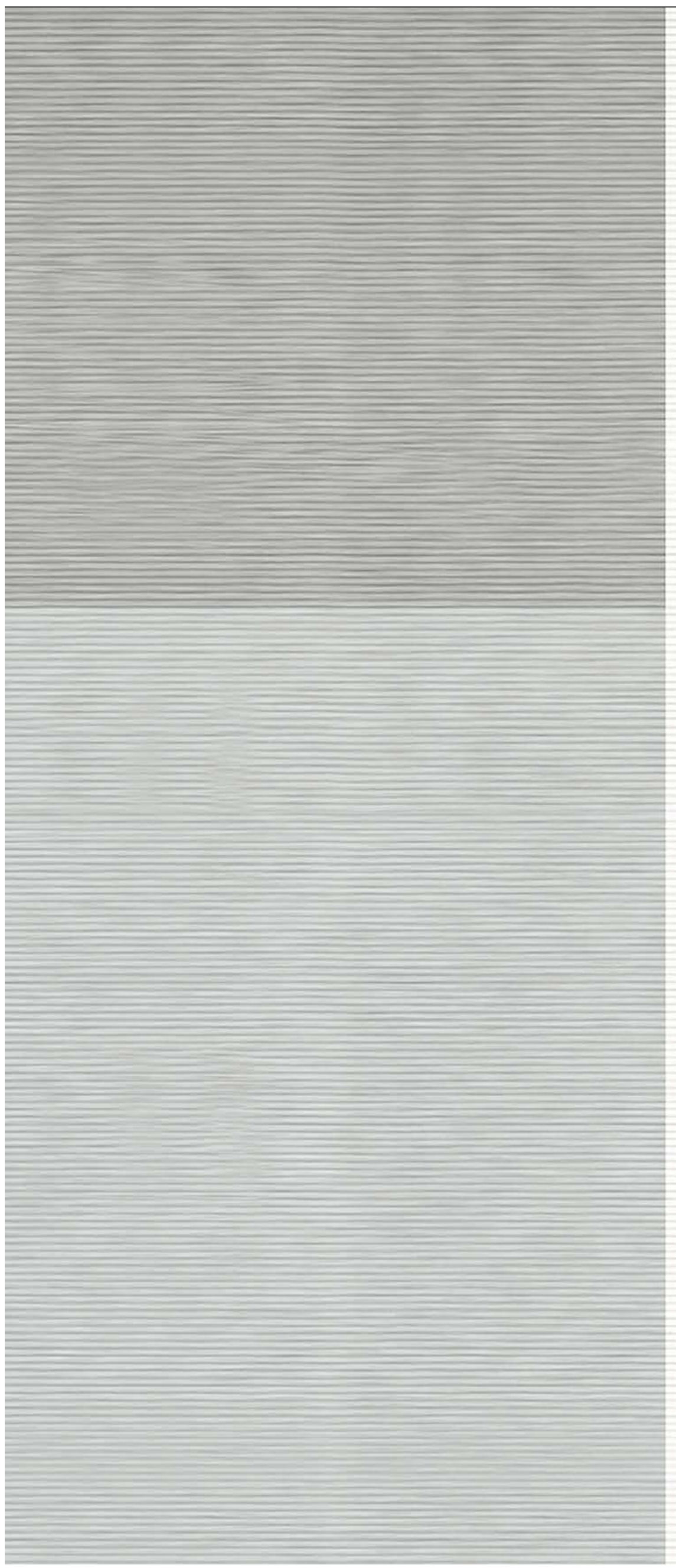

